\title{
Authors' reply: Comment on: Clinical implications of prompt ascitic drain removal in cirrhosis with refractory ascites
}

Singapore Med J 2021; 62(12): 666 https://doi.org/10.11622/smedj.2021237

Dear Sir,

We thank the author ${ }^{(1)}$ for his interest in our recent work demonstrating an increased risk of ascitic drain-related peritonitis and acute kidney injury in patients with cirrhosis requiring large-volume paracentesis. ${ }^{(2)}$ The author brought up the interesting concept of tunnelled long-term ascites drainage (LTAD), a cost-effective alternative to fulfil the palliative need in patients with malignant ascites. As the author rightly pointed out, the existing data for LTAD is preliminary. Current studies on LTAD are confounded by the small sample size and limited follow-up duration. While healthcare resource utilisation was reduced, the study failed to demonstrate an improvement in the quality of life of patients with LTAD, which is an important consideration when it comes to patient-centric palliative hepatology. ${ }^{(3)}$

Patient selection is the key. While LTAD may be an acceptable alternative in patients with malignant ascites who have very limited survival time (median survival was only 53 days in the LTAD group) ${ }_{1}^{(3)}$ we caution against extrapolating the benefit to all cirrhosis patients with refractory ascites, where the median survival is generally longer. In our current study, we demonstrated an increased risk of ascitic drain-related peritonitis and acute kidney injury in cirrhosis patients with refractory ascites when ascitic drain removal was delayed beyond 24 hours. Ascitic drain is an important risk factor for nosocomial spontaneous bacterial peritonitis, which is known to result in poorer survival among patients with decompensated cirrhosis. ${ }^{(4)} \mathrm{A}$ similar finding was demonstrated in a multicentre prospective study on the automated low-flow ascitic pump, where a high risk of bacterial infection was observed in $43.3 \%$ of patients. ${ }^{(5)}$ Although the bacterial infection may be partially mitigated by administering long-term antibiotics in these patients, the potential adverse effect of long-term antibiotic prophylaxis in these patients with LTAD remained unclear.

Finally, we agree with the author that more studies are required before tunnelled LTAD can be widely adopted in the management of refractory ascites. Long-term safety outcome, patient-reported outcomes and patients' preferences must be considered when selecting patients for LTAD.

Yours sincerely,

Yu Jun $\underline{\text { Wong }}^{1,2}$, Rahul $\underline{\text { Kumar }}^{1,2}$, Jessica $\underline{\operatorname{Tan}}^{1}$, Prem Harichander Thurairajah ${ }^{3}$

${ }^{1}$ Department of Gastroenterology and Hepatology, Changi General Hospital, Singapore, ${ }^{2}$ Duke-NUS Academic Medicine Programme, SingHealth, ${ }^{3}$ Department of Gastroenterology and Hepatology, National University Hospital, Singapore. Eugene.wong.y.j@singhealth.com.sg

\section{References}

1. Thakur K. Comment on: Clinical implications of prompt ascitic drain removal in cirrhosis with refractory ascites. Singapore Med J 2021; $62: 665$.

2. Wong YJ, Lum HM, Tan PT, et al. Clinical implications of prompt ascitic drain removal in cirrhosis with refractory ascites. Singapore Med J 2021 ; $62: 659$.

3. Macken L, Bremner S, Gage H, et al. Randomised clinical trial: palliative long-term abdominal drains vs large-volume paracentesis in refractory ascites due to cirrhosis. Aliment Pharmacol Ther 2020; 52:107-22

4. Wong YJ, Kalki RC, Lin KW, et al. Short- and long-term predictors of spontaneous bacterial peritonitis in Singapore. Singapore Med J 2020 ; $61: 419-25$.

5. Wong F, Bendel E, Sniderman K, et al. Improvement in quality of life and decrease in large-volume paracentesis requirements with the automated low-flow ascites pump. Liver Transpl 2020; 26:651-61. 